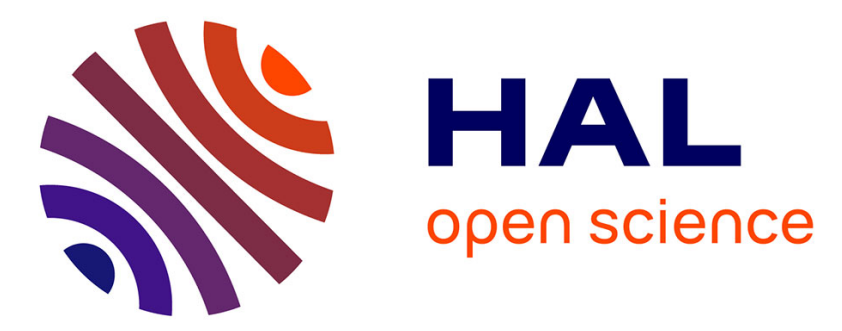

\title{
(Special Issue STEP-NC) Enabling feedback of process data by use of STEP-NC
}

\author{
Michael Wosnik, Christian Kramer, Andreas Selig, Peter Klemm
}

\section{To cite this version:}

Michael Wosnik, Christian Kramer, Andreas Selig, Peter Klemm. (Special Issue STEP-NC) Enabling feedback of process data by use of STEP-NC. International Journal of Computer Integrated Manufacturing, 2006, 19 (06), pp.559-569. 10.1080/09511920600622080 . hal-00513370

\section{HAL Id: hal-00513370 \\ https://hal.science/hal-00513370}

Submitted on 1 Sep 2010

HAL is a multi-disciplinary open access archive for the deposit and dissemination of scientific research documents, whether they are published or not. The documents may come from teaching and research institutions in France or abroad, or from public or private research centers.
L'archive ouverte pluridisciplinaire HAL, est destinée au dépôt et à la diffusion de documents scientifiques de niveau recherche, publiés ou non, émanant des établissements d'enseignement et de recherche français ou étrangers, des laboratoires publics ou privés. 


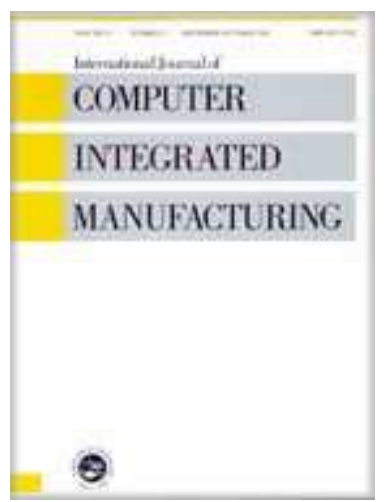

\section{(Special Issue STEP-NC) Enabling feedback of process data by use of STEP-NC}

\begin{tabular}{|c|c|}
\hline Journal: & International Journal of Computer Integrated Manufacturing \\
\hline Manuscript ID: & TCIM-2006-IJCIM-0017 \\
\hline Manuscript Type: & Special Issue Paper \\
\hline $\begin{array}{r}\text { Date Submitted by the } \\
\text { Author: }\end{array}$ & 01-Feb-2006 \\
\hline Complete List of Authors: & $\begin{array}{l}\text { Wosnik, Michael; Institute for Control Engineering of Machine Tools } \\
\text { and Manufacturing Units (ISW), University of Stuttgart } \\
\text { Kramer, Christian; Institute for Control Engineering of Machine } \\
\text { Tools and Manufacturing Units (ISW), University of Stuttgart } \\
\text { Selig, Andreas; Institute for Control Engineering of Machine Tools } \\
\text { and Manufacturing Units (ISW), University of Stuttgart } \\
\text { Klemm, Peter; Institute for Control Engineering of Machine Tools } \\
\text { and Manufacturing Units (ISW), University of Stuttgart }\end{array}$ \\
\hline Keywords: & CNC, PROCESS MONITORING, STEP NC \\
\hline Keywords (user): & servo drives, process force reconstruction \\
\hline
\end{tabular}

\section{S ScholaroNE" \\ Manuscript Central}


IESM-05 Special Issue

Catchline (head of first page only)

International Journal of Computer Integrated Manufacturing, Vol. X,

No. X, Month 2004, xxx-xxx

Running heads

(verso) M. Wosnik et al.

(recto) Feedback of process data by use of STEP-NC

Article Type (e.g. Review Article, Research Note, Brief Communication - if known)

\title{
Enabling feedback of process data by use of STEP-NC
}

MICHAEL WOSNIK*, CHRISTIAN KRAMER*, ANDREAS SELIG* and PETER KLEMM

ISW, University of Stuttgart, Seidenstr 36, Stuttgart, 70174, Germany, fax: +49-(0)711-121-2413

Correspondence *Corresponding authors. Email: Michael.Wosnik@isw.uni-stuttgart.de, phone: +49-(0)711-121-2342

Email: Christian.Kramer@isw.uni-stuttgart.de, phone: +49-(0)711-121-2433

Email: Andreas.Selig@isw.uni-stuttgart.de, phone: +49-(0)711-121-2464

\begin{abstract}
Today's Computer Aided Process Planning (CAPP) systems are performing NC programming with insufficient consideration of real machine capabilities and resulting workpiece characteristics. Computer Numerical Controls (CNCs) receive no information about actual workpiece characteristics and applied manufacturing processes. Machine and process observation are not closely coupled to the actual executed machining workingstep. STEP-NC addresses these problems by aiming at the availability of higher information content in CNCs. In this paper, a generic approach to preprocess and feed back process data from servo drives to CNCs and CAPP systems is presented. This approach rests on the application of open digital servo drives in machine tools and the design of application-dependent algorithms to process and exchange drive signals for both, online and offline optimisation of machining processes. The effects of the proposed methodology on design characteristics of open servo drives and CNC kernels as well as on the data interfaces and communication protocols between their modules and CAPP systems are focussed in this paper. The methodologies in this paper are exemplified by their application to drive-spanning process force reconstruction.
\end{abstract}

Keywords: CNC; servo drives; observer theory; process force reconstruction 


\section{Introduction}

Research in feature technology for manufacturing is primary focussed on various tasks in process planning, like feature recognition techniques, set-up planning, tool accessibility analysis, tool selection and operation sequencing (van Houten 1991, Shah and Maentylae 1995). However, machine-specific process characteristics are not automatically assigned to the respective features and thus are considered only to a limited extent in future planning tasks.

To address the lack of machine-optimality of control data and its processing in CNC machine tools, various kinds of methods and routines for data and processing optimisation with respect to machining time and quality considerations are offered in literature and commercial products.

Offline optimisation may be done by adjustment of a programmed feed rate in CNC control data (http://www.cgtech.com/optipath.htm [January 2005]) or other ways of control data refinement. If an optimised G \& M code (ISO 6983) CNC program has to short lines, intermittent cut may occur due to overload of the CNC kernel, which may be addressed by integration of feed rate adjustment modules into the CNC (Mori et al. 2001).

Extensive research has been dedicated to online process monitoring and control in the past 30 years, but adoption in commercial CNCs is still widely missing (Liang et al. 2004). Openness of supplied control systems is expected to offer machine tool manufacturers and end-users the possibility to integrate custom control functions (Pritschow et al. 2001, Meo 2004). Openness of CNCs and servo drives will ease use of process monitoring and control applications, which are usually installed as a separate hard-/software application communicating with the CNC by use of the Programmable Logic Controller (PLC) interface today (Brouer 2000, Schweiker 2003).

The low information content of $\mathrm{G} \& \mathrm{M}$ code hampers online adjustment of the machining process. It is only performed with limited consideration of the characteristics of the actual feature, the applied tool and the respective process type, since no appropriate information is available in common $\mathrm{G} \& \mathrm{M}$ code. To address the limited information availability on the shop floor and especially in the CNC, worker-oriented machining models have been developed in the middle of the1990s which combine manufacturing features and machining operations into machining objects (Storr et al. 1995). Since these machining models usually had to be converted to $\mathrm{G} \& \mathrm{M}$ code, the corresponding machining objects were available on Human Machine Interface (HMI) level only, but not transferred to the CNC kernel. Machining models which were proposed to be interpreted by the CNC kernel directly (Fechter 1996, Weck and Schulze 1996) based the STEP-NC standard (ISO 14649) and its correspondent STEP draft (ISO 10303-238). In the following, various architectures of STEP-NC-enabled CNCs were proposed and implemented (Suh et al. 2002, Lee and Bang 2003). In particular, Brouer (2000) and Schweiker (2003) developed general CNC kernel architectures for the integration of process monitoring and control modules embedded into open architecture CNCs with STEP-NC interpreters.

However, integration of algorithms for retrieval and processing of process data in open servo drives are not addressed extensively in recent approaches. Thus, this paper focuses on possibilities for the integration of application-specific algorithms for the retrieval of process data into digital open servo drives and the condensing, transferring and usage of process data in upstream CNC and CAPP systems.

\section{Usage of drive signals for process monitoring and optimisation}

Drive signals are usually used for drive-specific purposes, like the control of the corresponding axis movements and thus are already available in various kinds, like positions, speeds and motor currents. To increase the robustness of process monitoring and control and to minimise expenses for additional sensing devices, drive signals may be used as input for several process monitoring and control functions (Pritschow et 
al. 1999). Figure 1 summarises different types of monitored drive data usage, which may also be pursued simultaneously.

[Insert figure 1 about here]

In some cases of interest, drive signals may be evaluated straightforward for an adaptive control application. For instance, Brecher and Wolf (2004) presented an application for the feedback of the spindle torque signal to adapt the cutting width and cutting depth of pocket milling with respect to the appearance of chatter and implemented it into a STEP-NC-enabled CNC.

Since drive signals do not correspond directly to interesting process variables in general, in most cases application dependent algorithms have to be applied to process and analyse these signals. In general, modelbased and heuristic approaches, like the application of artificial neuronal networks (ANN), can be distinguished (Liang et al. 2004). To decrease the amount of experiments to gain the drive signal processing model, a model-backed observer approach is chosen.

\subsection{Estimation of process states by use of observers}

In cybernetics, observers are often used to estimate process variables of interest which are not directly observable, but are intended to be controlled. Observers require a model, which describes the interrelation of input variables, state variables and output variables. The following foundations of observer theory are used to implement the process state observers for the different machine tool drives and spindles.

In state space, a linear system is described by the following two equations:

$\dot{\mathbf{x}}(t)=\mathbf{A x}(t)+\mathbf{B u}(t)$

$\mathbf{y}(t)=\mathbf{C x}(t)+\mathbf{D u}(t)$

Here, the state vector is denoted as $\mathbf{x}(t)$, the known input vector is denoted as $\mathbf{u}(t)$ and the measurable output vector is denoted as $\mathbf{y}(t)$. The feedforward matrix $\mathbf{D}$ is in most cases a zero matrix.

A Luenberger observer (Luenberger 1964) may be used to estimate the state vector. The corresponding observer equations are:

$$
\begin{aligned}
& \dot{\hat{\mathbf{x}}}(t)=\hat{\mathbf{A}} \hat{\mathbf{x}}(t)+\hat{\mathbf{B}} \mathbf{u}(t)+\mathbf{H}[\mathbf{y}(t)-\hat{\mathbf{y}}(t)] \\
& \hat{\mathbf{y}}(t)=\hat{\mathbf{C}} \hat{\mathbf{x}}(t)+\hat{\mathbf{D}} \mathbf{u}(t)
\end{aligned}
$$

In equations (3) and (4), a circumflex remarks estimations for states, output variables and matrices.

In physical systems, states are usually foreseen for continuously changing variables, e.g. various energy forms or current in inductive systems. By modelling process variables as additional states without dynamics on their own, they may be estimated as well similar to a state-and-disturbance-observer (Weihrich 1978, Pritschow et al. 1999, Park and Altintas 2004), resulting in system (5), (6).

$$
\begin{aligned}
& \left(\begin{array}{c}
\dot{\mathbf{x}}(t) \\
\dot{\mathbf{x}}_{\mathbf{d}}(t)
\end{array}\right)=\left(\begin{array}{cc}
\mathbf{A} & \mathbf{C}_{\mathbf{d}} \\
\mathbf{0} & \mathbf{A}_{\mathbf{d}}
\end{array}\right)\left(\begin{array}{c}
\mathbf{x}(t) \\
\mathbf{x}_{\mathbf{d}}(t)
\end{array}\right)+\left(\begin{array}{c}
\mathbf{B} \\
\mathbf{0}
\end{array}\right) \mathbf{u}(t) \\
& \mathbf{y}(t)=\left(\begin{array}{ll}
\mathbf{C} & \mathbf{0}
\end{array}\right)\left(\begin{array}{c}
\mathbf{x}(t) \\
\mathbf{x}_{\mathbf{d}}(t)
\end{array}\right)+\mathbf{D u}(t),
\end{aligned}
$$

where $\mathbf{x}_{\mathbf{d}}(t)$ is the state vector for disturbances.

Reduced observers may be used in cases, where state variables can be measured directly or calculated from measurements, and thus do not need to be estimated. If the state variables can be grouped in a way that the transformation (7) is regular, a reduced observer for the state vector $\mathbf{x}_{2}(t)$ with dimension $(n-q)$ will be obtained. 
$\left(\begin{array}{c}\mathbf{y}(t)-\mathbf{D u}(t) \\ \mathbf{x}_{\mathbf{2}}(t)\end{array}\right)=\left(\begin{array}{cc}\mathbf{C}_{\mathbf{1}} & \mathbf{C}_{\mathbf{2}} \\ \mathbf{0} & \mathbf{I}_{\mathbf{n}-\mathbf{q}}\end{array}\right)\left(\begin{array}{l}\mathbf{x}_{\mathbf{1}}(t) \\ \mathbf{x}_{\mathbf{2}}(t)\end{array}\right)$

In case the matrices of the observer model are estimated adequately, i.e. $\hat{\mathbf{A}}$ is identical to $\mathbf{A}$ etc., the following differential equations for the estimation of the process states and for the observer error are obtained by combination of equations (1), (3), (4):

$\dot{\hat{\mathbf{x}}}(t)=\mathbf{A} \hat{\mathbf{x}}(t)+\mathbf{H}[\mathbf{y}(t)-\mathbf{C} \hat{\mathbf{x}}(t)]+(\mathbf{B}-\mathbf{H D}) \mathbf{u}(t)$

$\frac{d[\hat{\mathbf{x}}(t)-\mathbf{x}(t)]}{d t}=(\mathbf{A}-\mathbf{H C})[\hat{\mathbf{x}}(t)-\mathbf{x}(t)]$

The observer gain matrix $\mathbf{H}$ should be chosen by the observer designer in a way that the eigenvalues of the matrix $\mathbf{A}-\mathbf{H C}$ are lying left to the eigenvalues of the matrix $\mathbf{A}$ in the complex s-plane. However, too far left-placed eigenvalues are increasing the measuring noise of $\mathbf{y}(t)$.

\subsection{Application of drive signals for process force reconstruction}

Knowledge about process forces is an important aspect in process planning. The process force influences the dynamics of the machine tool with the danger of chatter and increased tool wear. In addition workpiece errors, e.g. due to tool deflection and chip flow, may be assigned to characteristic process force values.

For instance, approaches for the estimation of applied forces on spindles (Park and Altintas 2004), ball-screw-drives (Pritschow et al. 1999) and linear direct drives (Pritschow et al. 2004) were developed in the past, in each case related solely to investigated moving unit.

In the following, approaches of Pritschow et al. to drive-related process force reconstruction forming the base for the drive-spanning process force reconstruction, which is proposed in section 0, are recapitulated. Details regarding the effectiveness of these approaches may be taken from the relevant publications (Pritschow et al. 1999, Pritschow et al. 2004).

2.2.1 Ball-screw-drives. For a linear drive of a machine tool with rotary motor and a ball screw which is independently mounted on the machine tool frame, the following differential equation for the slide motion $x$ depending on the motor rotation $\varphi$ is premised (Pritschow et al. 1999):

$m \ddot{x}+d \dot{x}+c x+F_{f r i c}(\dot{x})=\frac{h_{s p}}{2 \pi i_{g}}(d \dot{\varphi}+c \varphi)+F_{p r o c}+F_{\text {const }}$,

where $m$ is the reduced mass of the slide, possibly containing the mass of carried additional drives, $c$ and $d$ are the parameters of a axis-parallel spring-damper system connecting the screw and nut of the ball screw, $h_{s p}$ is the pitch of the ball screw, $i_{g}$ is the transmission ratio of the drive gear, $F_{\text {fric }}$ results from the velocity-dependent friction of the guide slide bearing, $F_{p r o c}$ denotes the process force to be reconstructed and $F_{\text {const }}$ denotes components of constant forces, like gravitational force, in movement direction. The friction force is to assumed to depend non-linear on the slide speed solely. Thus, load-dependent friction, e.g. on force components resulting from the process or carried slides, is neglected in this analysis. The position-varying dynamic behaviour of the rest of the drive chain is also neglected.

By measuring motor rotational speed and position as well as slide velocity and position, equations (5) and (6) lead to: 


$$
\begin{aligned}
& \dot{\mathbf{x}}(t)=\left(\begin{array}{ccc}
0 & 1 & 0 \\
-c / m & -d / m & -1 / m \\
0 & 0 & 0
\end{array}\right) \mathbf{x}(t)+\frac{h_{s p}}{2 \pi i i_{g} m}\left(\begin{array}{ll}
0 & 0 \\
c & d \\
0 & 0
\end{array}\right) \mathbf{u}(t) \\
& \mathbf{y}(t)=\left(\begin{array}{ccc}
1 & 0 & 0 \\
0 & 1 & 0
\end{array}\right) \mathbf{x}(t), \\
& \mathbf{x}(t)=\left(\begin{array}{c}
x(t) \\
\dot{x}(t) \\
F_{\text {fric }}(t)-F_{\text {proc }}(t)-F_{\text {const }}
\end{array}\right), \\
& \mathbf{u}(t)=\left(\begin{array}{c}
\varphi(t) \\
\dot{\varphi}(t)
\end{array}\right), \\
& \mathbf{y}(t)=\left(\begin{array}{c}
x(t) \\
\dot{x}(t)
\end{array}\right) .
\end{aligned}
$$

Since the measured output directly corresponds to a part of the state vector, i.e. the transformation (7) required is the identity matrix, a reduced observer is obtained straightforward. Thus, (8) leads to:

$\dot{\hat{x}}_{r}=\frac{h_{12}}{m} \hat{x}_{r}-\left(\frac{h_{12} c h_{s p}}{2 \pi i_{g} m} \frac{h_{12} d h_{s p}}{2 \pi i_{g} m}\right) \mathbf{u}+\left(\frac{h_{11} h_{12}+c h_{12}}{m} \frac{h_{12}^{2}+d h_{12}}{m}\right) \mathbf{y}$,

where $\left(\begin{array}{ll}h_{11} & h_{12}\end{array}\right)$ denotes the reduced observer gain matrix.

The relation between the friction force and the slide speed has to be examined for each drive initially. The process force can be calculated according to (17).

$\hat{F}_{\text {proc }}=F_{\text {fric }}(\dot{x})-F_{\text {const }}-\hat{x}_{r}-\left(\begin{array}{ll}h_{11} & h_{12}\end{array}\right) \mathbf{y}$

2.2.2 Linear direct drives. For linear direct drives, i.e. drives without gears and ball screws, a single differential equation for an electro-mechanical system is obtained (Pritschow et al. 2004):

$m \ddot{x}+F_{\text {fric }}(\dot{x})=k_{m} i_{m}+F_{\text {proc }}+F_{\text {const }}$,

where $k_{m}$ is the motor constant and $i_{m}$ is the motor current, in addition to the descriptions of (10).

Motor movement signals of linear direct drives correspond directly to the slide movements, since no transmitting elements are present. The velocity quantisation can be decreased by integration of a directly measured acceleration signal instead of differentiating the position signal leading to advantages in ordinary drive operation (Pritschow et al. 2003). Since the Ferraris sensor measures relative accelerations, it may be used for drives carried by the frame as well as for drives carried by other drives.

The corresponding system is:

$$
\begin{aligned}
\dot{\mathbf{x}}(t) & =\left(\begin{array}{ccc}
0 & 0 & 1 \\
0 & 0 & 0 \\
0 & -1 / m & 0
\end{array}\right) \mathbf{x}(t)+\left(\begin{array}{c}
0 \\
0 \\
\frac{k_{m}}{m}
\end{array}\right) u(t) \\
\mathbf{y}(t) & =\left(\begin{array}{ccc}
1 & 0 & 0 \\
0 & -1 / m & 0
\end{array}\right) \mathbf{x}(t)+\left(\begin{array}{c}
0 \\
\frac{k_{m}}{m}
\end{array}\right) u(t),
\end{aligned}
$$


where

$$
\begin{aligned}
& \mathbf{x}(t)=\left(\begin{array}{c}
x(t) \\
F_{\text {fric }}(t)-F_{\text {proc }}(t)-F_{\text {const }} \\
\dot{x}(t)
\end{array}\right), \\
& u(t)=i_{m}(t), \\
& \mathbf{y}(t)=\left(\begin{array}{l}
x(t) \\
\ddot{x}(t)
\end{array}\right) .
\end{aligned}
$$

This leads to the following reduced observer:

$\dot{\hat{x}}_{r}=-h_{11} \hat{x}_{r}-h_{11}^{2} x+\left(h_{11} h_{12}+1\right) \ddot{x}-h_{11} h_{12} \frac{k_{m}}{m} i_{m}$

$\hat{v}=\hat{x}_{r}+h_{11} x+h_{12}\left(\ddot{x}-\frac{k_{m}}{m} i_{m}\right)$

$$
\hat{F}_{\text {proc }}=F_{\text {fric }}(\hat{v})-F_{\text {const }}+m \ddot{x}-k_{m} i_{m}
$$

Since the eigenvalues of the matrix $\mathbf{A}-\mathbf{H C}$ are independent from $h_{12}$, this element of the observer gain matrix may be set to zero. The process force may be calculated from (18) immediately, if an velocity signal is available, i.e. through integration from the acceleration signal. Measured process forces are compared to the reconstructed process forces in Figure 2 for a differently notated observer system concluding to the same equations, leading to a time delay of $3 \mathrm{~ms}$ for a $100 \mathrm{~Hz}$ shaker force introduction.

[Insert figure 2 about here]

2.2.3 Drive-spanning process-force estimation. To receive the drive-spanning process force, the process forces reconstructed from the several axes' drive signals have to be superposed, e.g. by use of the principle of virtual displacement.

If all drive-related forces have been calculated this way, for decoupled Cartesian machine tool kinematics, i.e. orthogonal machine axes, the drive-spanning process force is easily obtained by vector addition. The calculation of the process forces is more complicated if a machine tool drive axis is carrying a non-orthogonal slide. Then, additional friction forces depending on the relative movement of the carried slide may affect the dynamic equations (10) or (18), and thus require input of carried slide's velocities. In this case, the estimated process forces of (17) and (26) have to be modified in the CNC by use of fed back friction forces of carried guide slide bearings. Hence, the machine tool kinematics are not only required for the calculation of the drive-spanning process force, but also for the calculation of the drive-related process force components.

In case of interpolation of $\mathrm{X}-, \mathrm{Y}$ - and Z-axis, the drive-spanning process force is calculated to:

$\hat{\mathbf{F}}_{\text {proc }}\left(t_{i}\right)=\left(\begin{array}{c}\hat{F}_{\text {proc }}^{x}\left(t_{i}\right) \\ \hat{F}_{\text {proc }}^{y}\left(t_{i}\right) \\ \hat{F}_{\text {proc }}^{z}\left(t_{i}\right)\end{array}\right)$,

where the high indices denote the corresponding drive of the process force component for every sampled time increment $t_{i}$. In general, milling feed forces and cutting forces are superposed. The Y-axis process force component in two-axle turning, corresponding to the cutting force, may be measured by use of a spindle observer, which is not discussed in this paper, or by use of a observer for the Y-axis drive, if present. 
2.2.4 Feedback of process forces for process planning. In a multi-purpose machining environment, fed back process data has to linked to the context of their retrieval. This context is not provided by standard $\mathrm{G} \& \mathrm{M}$ code. Even the type of the machining process is not known, if subroutines are not applied. Thus, process data fed back from CNCs to CAPP systems have to be linked to a machining model, which is supplied by a STEPNC.

A schema for actual process data containing references to STEP-NC data elements is designed and detailed for the feedback of process forces (Figure 3 and Figure 4), where the number blocks, e.g. 4, 17 or 4, 17 (3), represent stubs for entity references.

[Insert figure 3 about here]

The usage of fed back process forces may require several measures for process forces, which may be done by an entity similar to the motor_current_observer. It is necessary to feed back drive-related signals to estimate statically and dynamically critical force values. Regarding the interaction between cutting tool and workpiece, drive-spanning process forces with respect to the contact zone between cutting tool and workpiece are required. Thus, several context data elements are required. To reuse process data in future planning situations, fed back process data should be

- related to the machine tool on which the corresponding process was performed, to the cutting tool which was applied, and to the time when it was gained,

- related to the definition of the workingstep, i.e. the feature to be manufactured and the operation characteristics which were designated,

- given in the workingstep coordinate system, which is usually the feature coordinate system. [Insert figure 4 about here]

This information is hold in the generic entities executed_workingstep and machined_workingstep_process_data of the process data schema. Since process data are usually not interchanged between different companies and are very diverse, a standardised process data model is neither required nor useful here. Thus, the entity machined_workingstep_process_data is foreseen to built an abstract supertype for arbitrary process data entities, which are exemplified in Figure 4. Since most process data are given as an array of values, the sampling rate has to be decreased in order to limit the amount of data exchanged for a single workingstep, and thus the progression of the force has to be averaged in a sampling period before feeding back the reconstructed forces.

By comparing fed back process force profiles to process forces obtained in previous machining situations with similar machining objects, process force calculation models may be updated, and machining parameters may be optimised.

\section{CNC and servo drive architecture}

In the long term, it is envisaged that all systems involved in the CAD/NC process chain are defining specific views on the same data base (Figure 5).

[Insert figure 5 about here]

The machine tool, with its CNC and servo drives, forms the critical part in the process chain regarding data availability and data processing speed requirements today, in normal case leading to individual configurations of few monolithic components. In order to achieve the attachment of process data to executed workingsteps, plain $\mathrm{G} \& \mathrm{M}$ code control data have to be replaced by data in a more encapsulated manner, preferably by use of STEP-NC as direct or as bidirectional convertible data input format. To relieve the CNC from extensive calculations with data supplied in interpolation rate, axis-specific control functions should be integrated in open digital servo drives, whereas axis-spanning functions have to be integrated in the $\mathrm{CNC}$ requiring feedback of condensed information from the drives. The use of STEP-NC poses new requirements 
on the CNC regarding data interpretation and linking of gained process data to interpreted control data, which are discussed in the following subsection.

\subsection{STEP-NC-enabled CNC}

STEP-NC may be used in several ways as direct or indirect input data format of CNCs. STEP data exchange requires extensive, non-deterministic communication and interpretation effort already for models which are not formally mapped to the Integrated Resources of STEP. On the other hand, CNCs have strong requirements on realtime capability for an important portion of their functionality, especially the interpolation and the data supply of the servo drives. Thus, various types of STEP-NC processing and downstream CNC functions have to be investigated.

The easiest way to weave the CNC into a STEP-NC-based CAD/NC process chain is to postprocess the machining model of STEP-NC to the standard or proprietary CNC input data format offline, preferably on the HMI operating unit of the CNC. In case no references to the original STEP-NC data objects is brought into the resulting $\mathrm{G} \& \mathrm{M}$ code control data, e.g. by use of standardised comments, attachment of process data to machining objects is cumbersome, online adaptation of the machining process with respect to the actual machining object is inhibited at all.

Increased encapsulation of machining objects is achieved by the use of subroutines (Figure 6), which are encompassing the machining object's parameterisation. In addition, functions for the retrieval of process data may be invoked. For the definition of subroutines, there are in general two possibilities:

- Compiled subroutines: Compiled subroutines are programmed in an advanced programming language, e.g. $\mathrm{C}++$, and require a development environment for linkage into the respective $\mathrm{CNC}$. Thus, they are usually only utilised by the $\mathrm{CNC}$ vendor or the machine tool manufacturer.

- $\mathrm{G} \& \mathrm{M}$ code subroutines: $\mathrm{G} \& \mathrm{M}$ code subroutines are programmed in the input data format of the control and thus may be edited on the HMI. Due to the loose integration with the CNC kernel, receiving and processing of process data is less comfortable compared to the application of compiled subroutines.

Process-oriented functions to fill user-defined data blocks, e.g. for reconstructed process forces to be fed back into the machining model by use of the process data schema, are enabled by the integration of corresponding applications. For CNCs without openness of CNC function blocks (Figure 6), the PLC interface may be utilised by the machine tool manufacturer to receive process data. An application for logging and further processing of preprocessed drive signals, which is integrated into the PLC, is limited by the cycle time and the data receiving requirements of the PLC. To overcome the shortcomings of this approach, open CNC kernels (Figure 7) with at least interpreter exchangeability, event submission and the possibility to integrate new functions are required.

[Insert figure 6 about here]

[Insert figure 7 about here]

The CNC shown in Figure 7 is able to process feature based process data. In order to do this, three non-realtime tasks have to be added to a today's conventional CNC and two realtime tasks have to be adapted.

Before realtime interpretation can be performed, the STEP-NC programs have to be interpreted and translated into enhanced $\mathrm{G} \& \mathrm{M}$ code, which is done by the postprocessing function block. This code must contain information about the actual machining object to be manufactured. We prefer an eXtensible Markup Language (XML)-like representation using opening and closing tags that are placed in the comment lines of the $\mathrm{G} \& \mathrm{M}$ code describing start and end of the workingsteps.

The interpretation task must be adapted to understand the new enhanced G \& $\mathrm{M}$ code and pass the information provided by it to the following tasks. The interpolation task must be extended so it can read and 
process the opening and closing tags in the enhanced $\mathrm{G} \& \mathrm{M}$ code and store it into an internal variable of the CNC.

The non-realtime tasks to be added beside the already described postprocessing of the STEP-NC programs are the processing and the supplying of the data provided by the drives via the fieldbus, e.g. SERCOS interface. In the processing task the process forces that result from a multidimensional movement are reconstructed. The supplying task passes the information to the CAPP system and the postprocessing task. In the postprocessing task this information is used to optimise further interpretation of STEP-NC-programs.

\subsection{Open digital servo drives}

Essential for servo drives used for preprocessing and extracting of drive signals is the openness of the drive controller. This openness is supported by the application of standardised communication interfaces, e.g. SERCOS interface, and a freely programmable servo drive based on Field Programmable Gate Arrays (FPGAs), which facilitate the machine tool manufacturer to integrate specific control algorithms as well as to adapt signal processing and exchange.

In contrast to DSP-based controllers whose interfaces are fixed and whose processors usually hold not enough resources for user specific algorithms, an FPGA-based system can be extended in software and hardware without great efforts. The controller can perfectly be adjusted to the needs of a specific process or machine. Open and clearly defined interfaces enable the user to include his own sensors and functionalities. He does not depend on the manufacturer of the drive controller and can react to the needs of his customers more quickly and flexibly.

But in order to achieve an open and flexible system which is easy to program and maintain, it is necessary to develop a comprehensive concept. The interfaces in hardware and software must be clearly defined. Mechanisms to maintain functionality and security of the whole system must be established. Furthermore, an easily usable developer environment with a library of standard functionalities has to be provided. A concept covering these issues is introduced in the following. The concept distinguishes three layers in which openness is offered as shown in Figure 8.

The very first layer is the hardware containing the programmable logic device. Extensions can be integrated as hardware submodules. In this layer a physical bi-directional interface to the CNC, like SERCOS interface can be integrated. The second layer resides on the programmable logic device. It is programmed with a hardware description language (e.g. VHDL, Verilog). Functionalities, like the algorithms needed by STEP-NC can be added as logic blocks. On the FPGA complete processors (CPUs and DSPs) can be programmed. They provide a third layer, on which functionalities can be added in standard programming languages (e.g. ASM, C, C++). Already existing drivers for SERCOS interface programmed in C can be compiled and loaded onto a CPU function block. This drive architecture enables the manufacturer to provide all functionalities needed by STEP-NC, including bi-directional communication. It is also possible to adjust the controller to the needs of special machines and processes.

[Insert figure 8 about here]

\subsection{Digital drive interface}

Today's digital servo drive interfaces are able to return actual position, speed, motor current and user-defined data in sampling rates which conform to CNC interpolation rates (http://www.sercos.de/pdf/SERCOSIII_WhitePaper_v134e.pdf [January 2005]).

Thus, it is possible to transport user defined data such as the process forces calculated in the drives in realtime into the CNC. In order to do so, user-specific parameters have to be defined in the SERCOS 
interface. These so-called P-parameter are used to address the data and to configure the drive to pass the information to the level above.

\section{Conclusions}

In this paper, a STEP-NC architecture from open digital servo drive over CNC to CAPP to process, transfer and evaluate process data was presented. The required extensions of today's CNCs and servo drives were referred. The reconstruction of process forces was used as an introducing example for the allocation of data processing and the resulting communication methods.

Today's CNCs and servo drives are lacking the openness required for the seamless implementation of the proposed methodology hampering the end-user of the machine tool to implement especially drive-related algorithms. In addition, usage of fed-back drive signals beyond visualisation of process force profiles require extensive reasoning on the context of machining and the most relevant factors for a distinct situation. There is much need of additional research in this field.

It is affordable to condense process force data further in the CNC, e.g. to medium cutting and feed forces, before feeding back to CAPP systems to decrease the enormous data exchange necessary to transfer STEP-NC entities, for instance in an ISO 10303-21 file. Further work has to be done to implement the CNC structures for drive-spanning process force reconstruction and the extended STEP-NC interface. The accuracy of the drive-related process force reconstructions may be increased by sophistication of stiffness-damping models leading the multi-spring-damper systems and to the integration of finite element portions of modelled components. However, these refinements lead to extensions of the dimension of the state vector and to increased calculation effort.

\section{References}

Brecher, C., Wolf, J., Prozessabhaengige Bahnplanung beim Taschenfraesen. wt Werkstattstechnik online, 2004, 94(7/8), 350-354 [in German].

Brouer, N., NC-Steuerungskern mit Datenschnittstelle für eine Autonome Produktionszelle, $\mathrm{PhD}$ thesis, Rheinisch-Westfaelische Technische Hochschule, 2000 [in German].

Fechter, T. A., Semantisches Modell zur Bearbeitungsbeschreibung fuer eine nutzergerechte Werkzeugmaschinensteuerung, $\mathrm{PhD}$ thesis, Technische Hochschule Darmstadt, Darmstadt, 1996 [in German].

Lee, W., Bang, Y.-B., Design and implementation of an ISO14649-compliant CNC milling machine. International Journal of Production Research, 2003, 41(13), 3007-3017.

Liang, S. Y., Hecker, R. L., Landers, R. G., Machining process monitoring and control: the state of the art. Journal of Manufacturing Science and Engineering, 2004, 126(2), 297-310.

Luenberger, D. G., Observing the state of a linear system. IEEE Transactions on Military Electronics, 1964, $\mathbf{8}(2), 74-80$.

Meo, F., Open controller enabled by an advanced real-time network (OCEAN). Proceedings of the $4^{\text {th }}$ CIRP International Seminar on Intelligent Computation in Manufacturing Engineering (CIRP ICME '04), Sorrento, Italy, 30 June - 2 July (Fisciano: CUES), 2004, pp. 589-594.

Mori, M., Yamazaki, K., Fujishima, M., Liu, J., Furukawa, N., A study on development of an open servo system for intelligent control of a CNC machine tool. CIRP Annals, 2001, 50(1), 247-250.

Park, S. S., Altintas, Y., Dynamic compensation of spindle integrated force sensors with Kalman filter. Journal of Dynamic Systems, Measurement, and Control, 2004, 126(3), 443-452.

Pritschow, G., Bretschneider, J., Fritz, S., Reconstruction of process forces within digital servodrive systems. Production Engineering, 1999, VI(1), 73-78. 
Pritschow, G., Altintas, Y., Jovane, F., Koren, Y., Mitsuishi, M., Takata, S., van Brussel, H., Weck, M., Yamazaki, K., Open controller architecture - past, present and future. CIRP Annals, 2001, 50(2), 463470.

Pritschow, G., Eppler, C., Lehner, W.-D., Ferraris sensor - the key for advanced dynamic drives. CIRP Annals, 2003, 52(1), 289-292.

Pritschow, G., Fritz, S., Pruschek, P., Reconstruction of process forces of direct drives using the Ferraris sensor. Proceedings of the VIIth International Conference on Monitoring and Automatic Supervision in Manufacturing AC 04, Zakopane, Poland, 19 - 21 August 2004, 2004.

Schweiker, A., Offene numerische Steuerungen fuer prozessabhaengige Bearbeitungen - vereinheitlichte Struktur, Funktionen und Schnittstellen, PhD thesis, Universitaet Stuttgart, 2003 [in German].

Shah, J. J., and Maentylae, M., Parametric and feature-based CAD/CAM: concepts, techniques and application, 1995 (New York: Wiley).

Suh, S. H., Cho, J. H., Hong, H. D., On the architecture of intelligent STEP-compliant CNC. International Journal of Computer Integrated Manufacturing, 2002, 15(2), 168-177.

Storr, A., Handel, D., Itterheim, C., Rommel, B., Stroehle, H., Manufacturing objects as uniform NC-interface for machining and measuring. Proceedings of the IFIP international conference on life cycle modelling for innovative products and processes (PROLAMAT '95), Berlin, Germany, 29 November - 1 December 1995, 1995 (London: Chapman \& Hall), pp. 356-368.

van Houten, F. J. A. M., PART: A computer aided process planning system. PhD thesis, Universiteit Twente, Enschede, 1991.

Weck, M., Schulze, O., A new CNC programming interface. Production Engineering, 1996, III(2), 101-104.

Weihrich, G., Drehzahlregelung von Gleichstromantrieben unter Verwendung eines Zustands- und Stoergroessenbeobachters. Regelungstechnik, 1978, 26(11), 349-380 [in German]. 
Figure captions

Figure 1. Types of drive signal usage.

Figure 2. Measured and observed process force of the reduced observer system for the linear direct drive [Source: Pritschow et al. 2004].

Figure 3. EXPRESS-G of process data model, excerpt of page 3.

Figure 4. EXPRESS-G of process data model, page 4.

Figure 5. STEP-NC reference architecture.

Figure 6. Usage of G \& M code subroutines and the PLC interface.

Figure 7. Architecture and interfaces of open STEP-NC-enabled CNC.

Figure 8. Three layers of openness. 


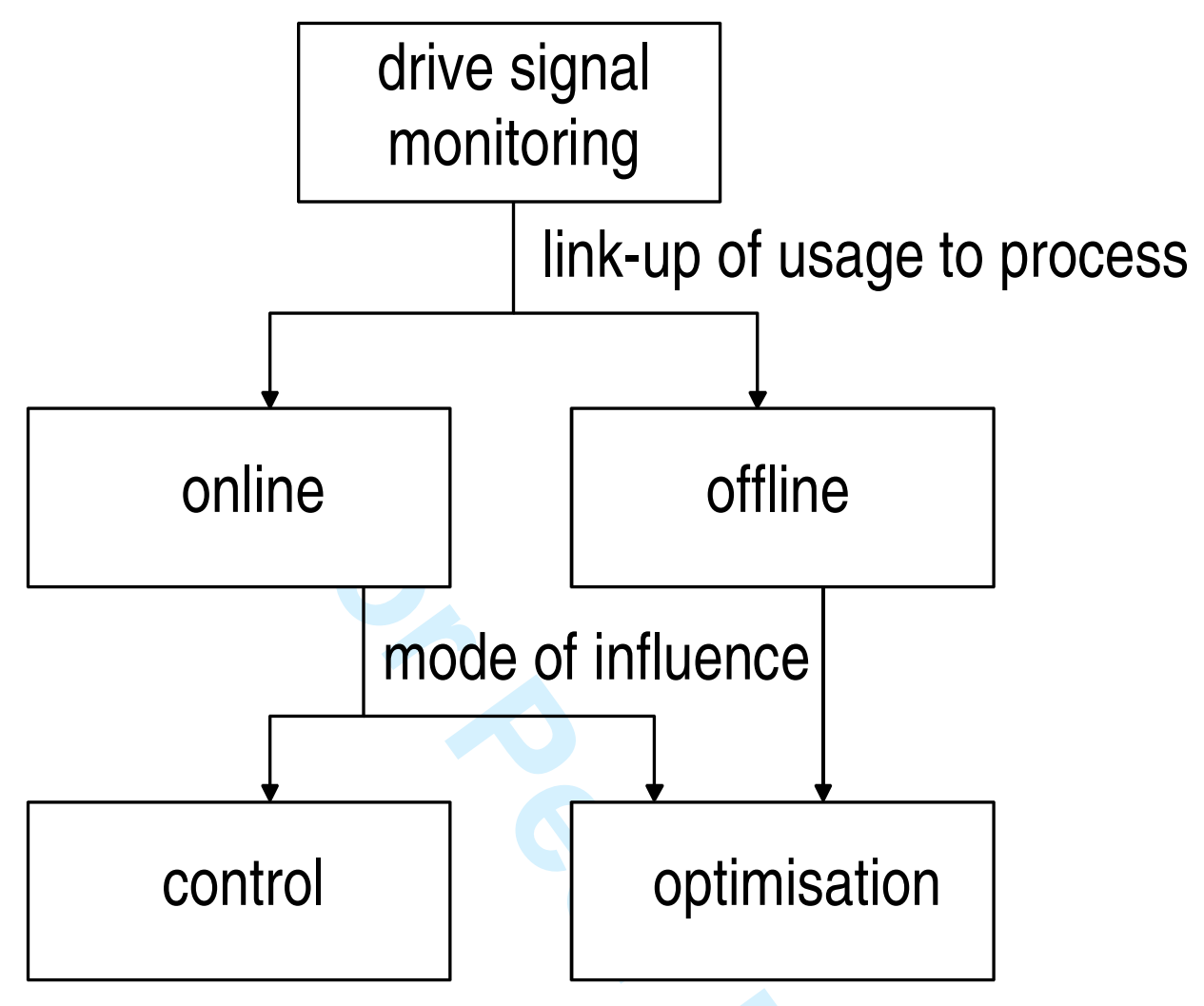



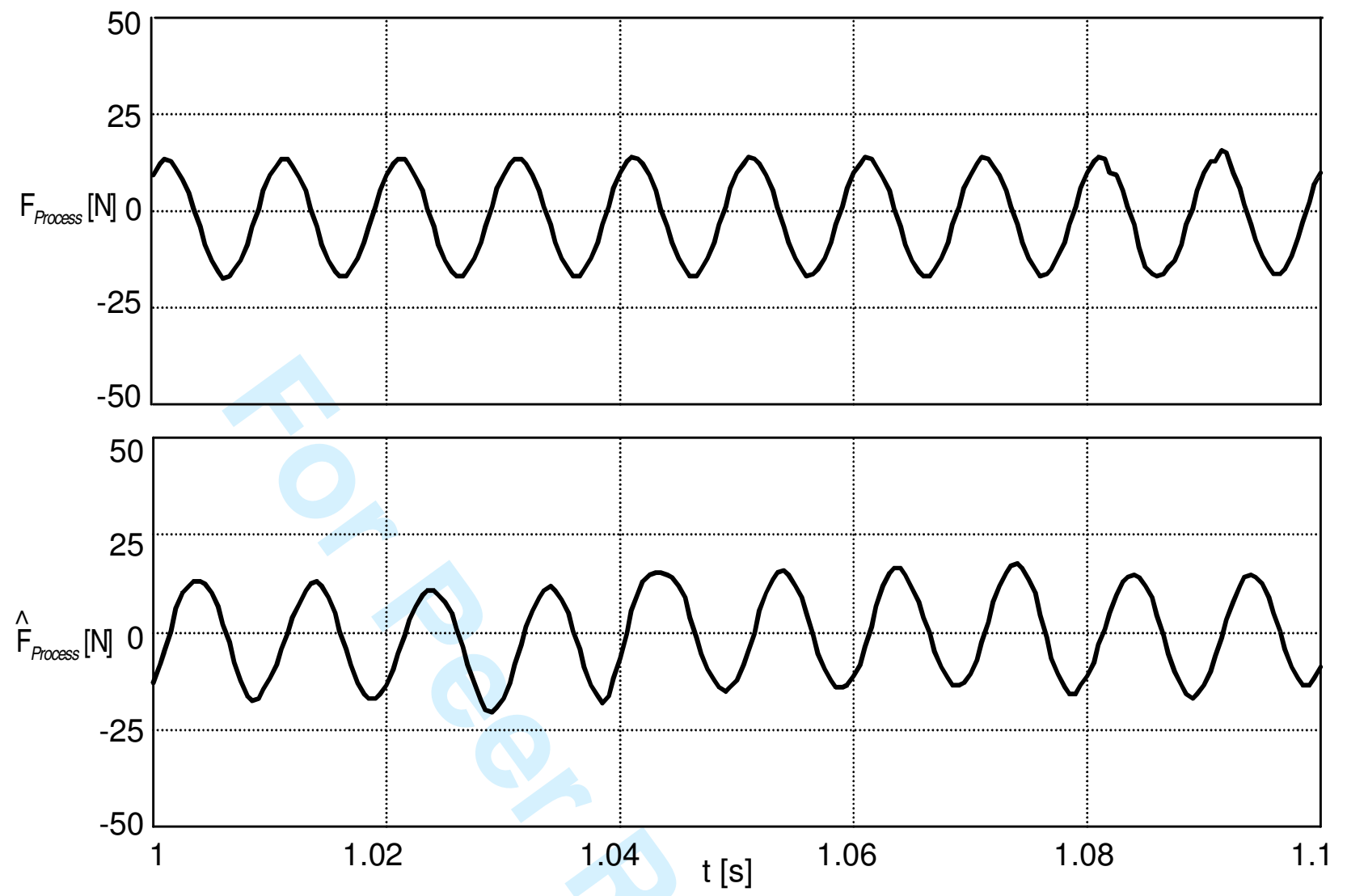


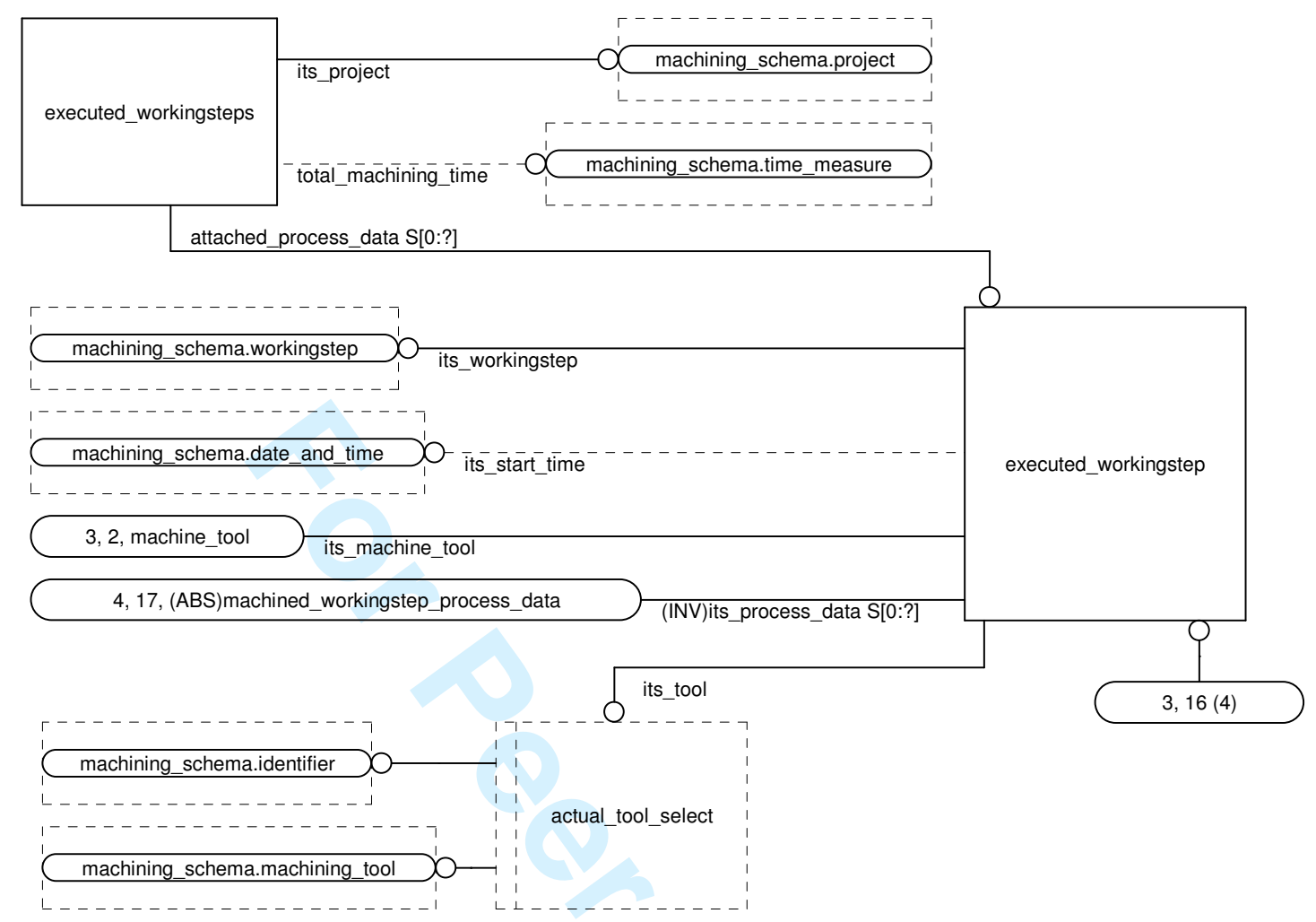




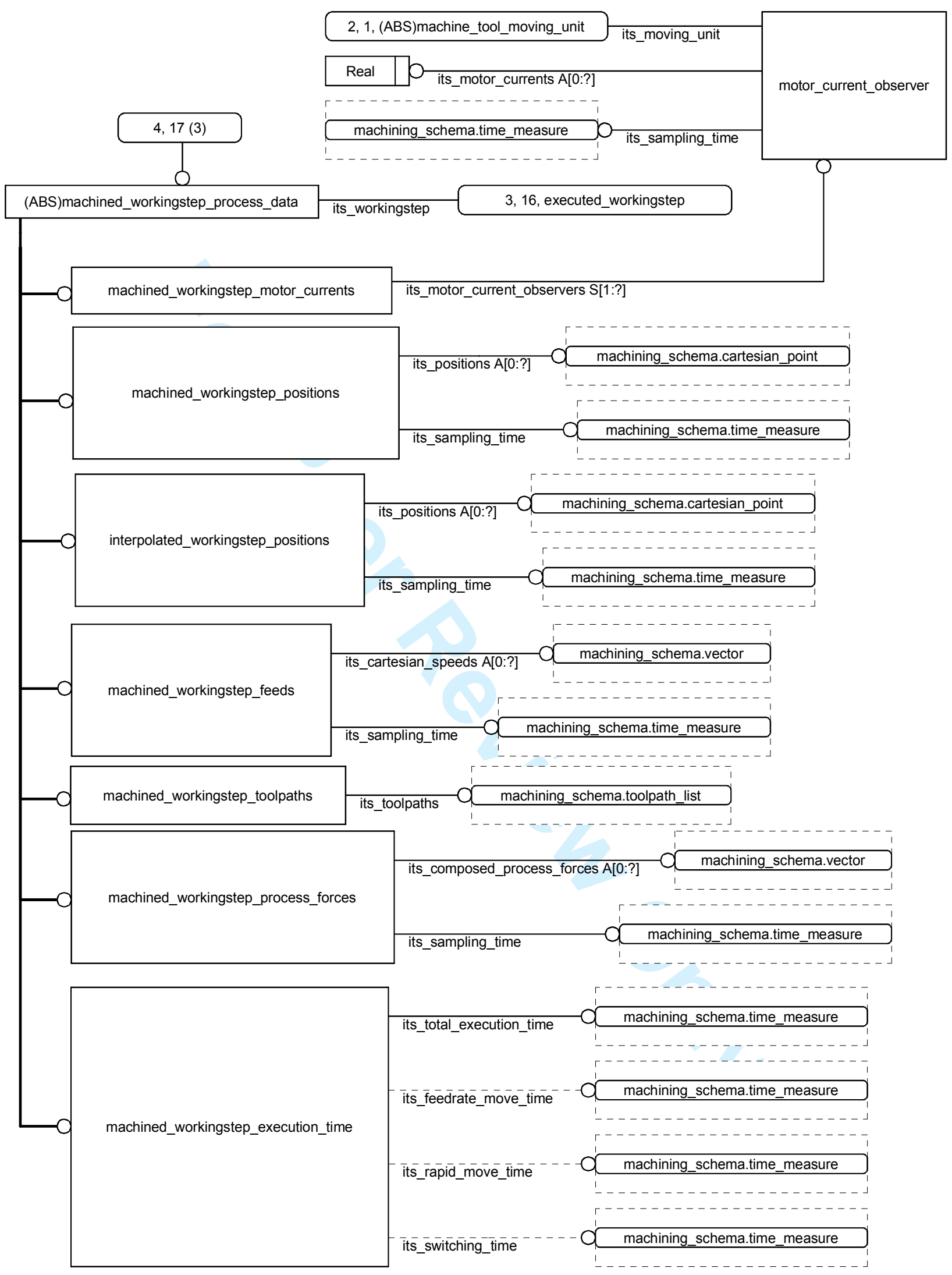




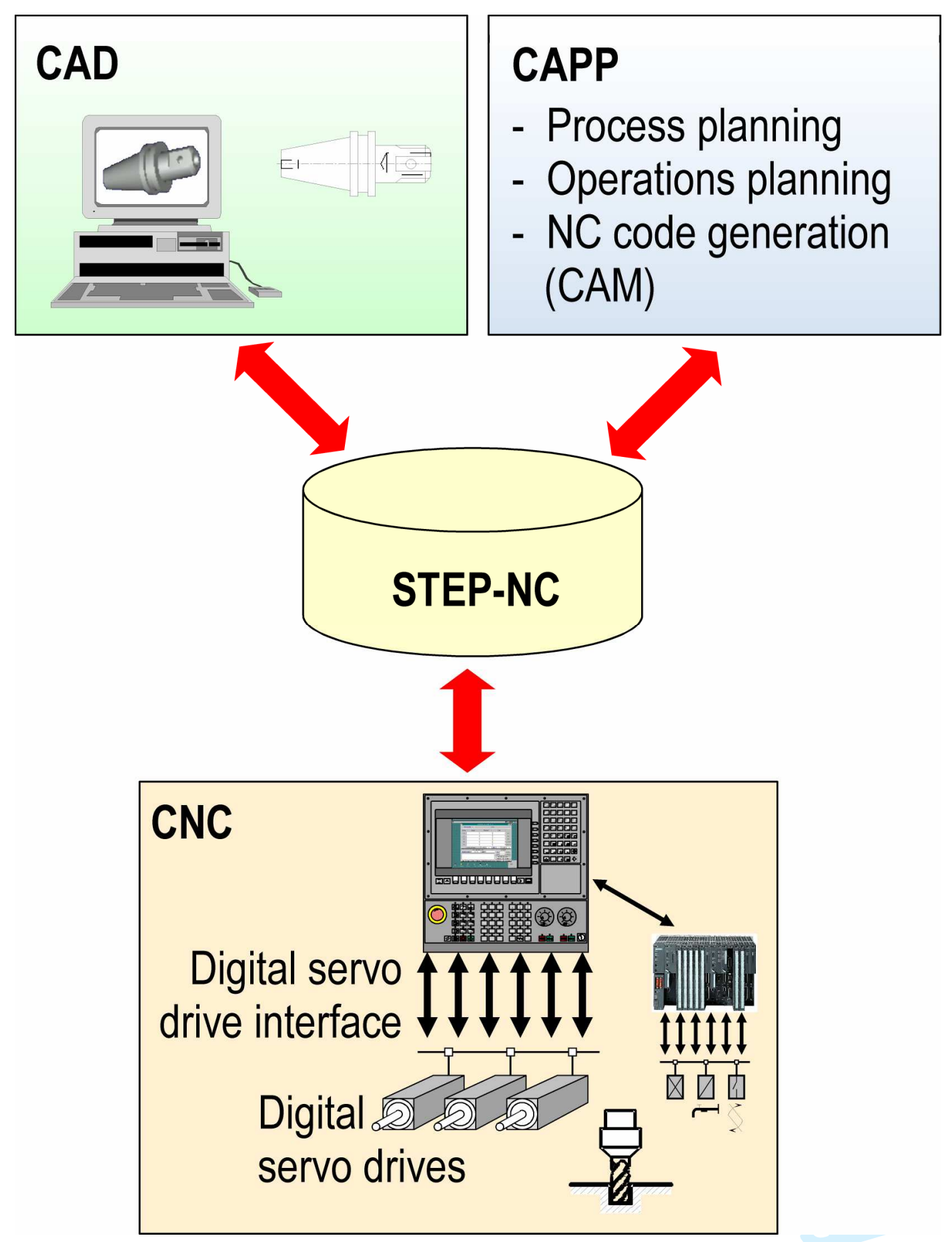




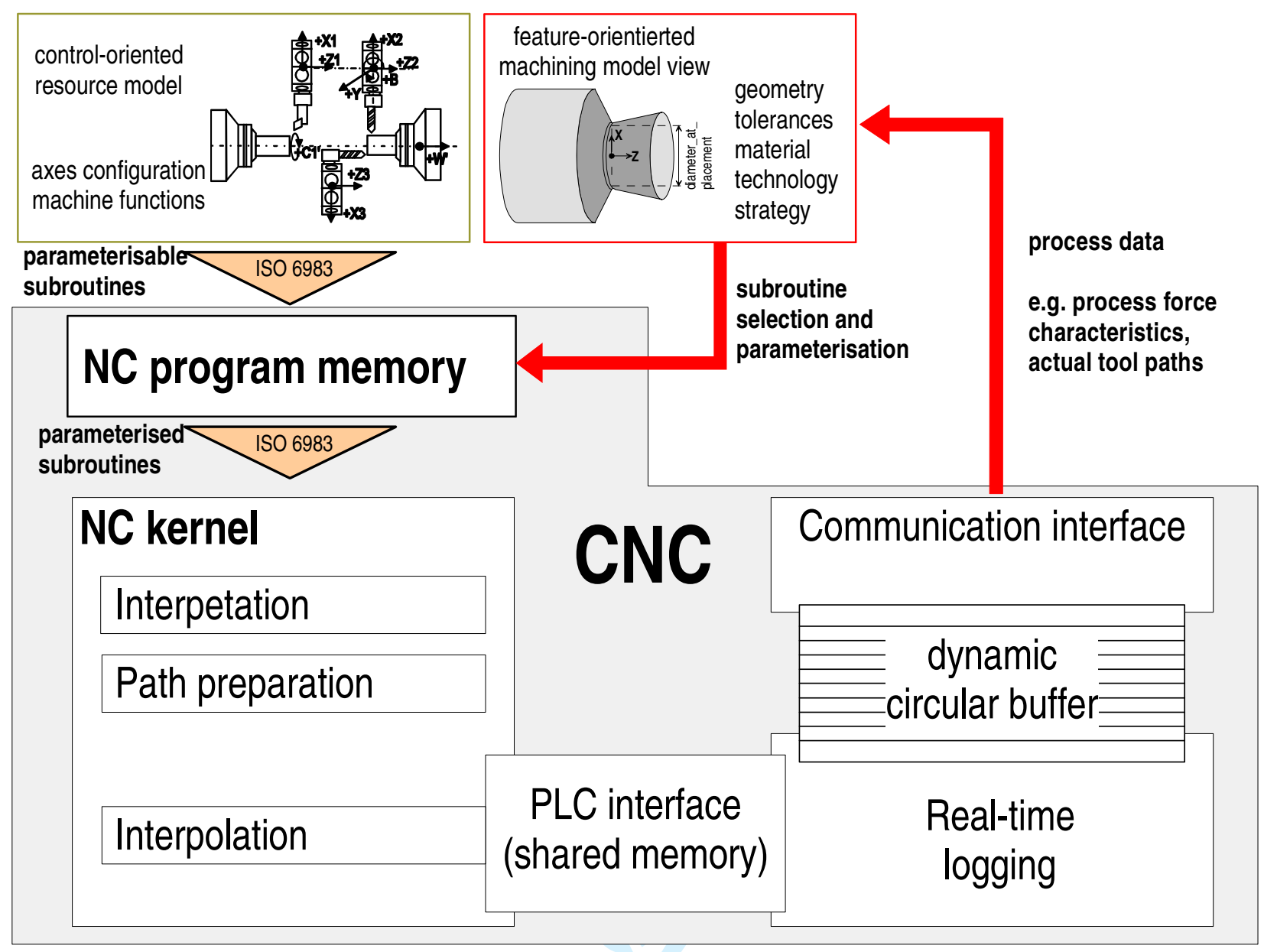




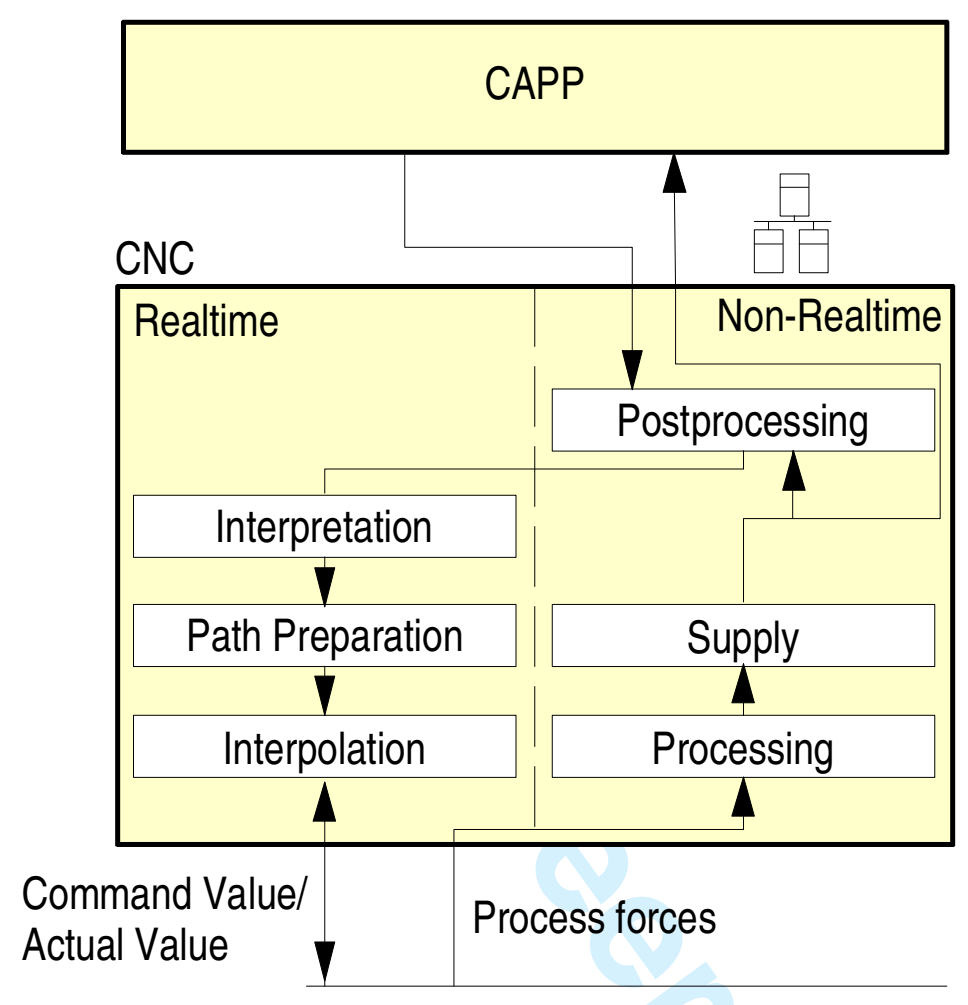

Fieldbus e.g. SERCOS 
3rd Layer:

CPU

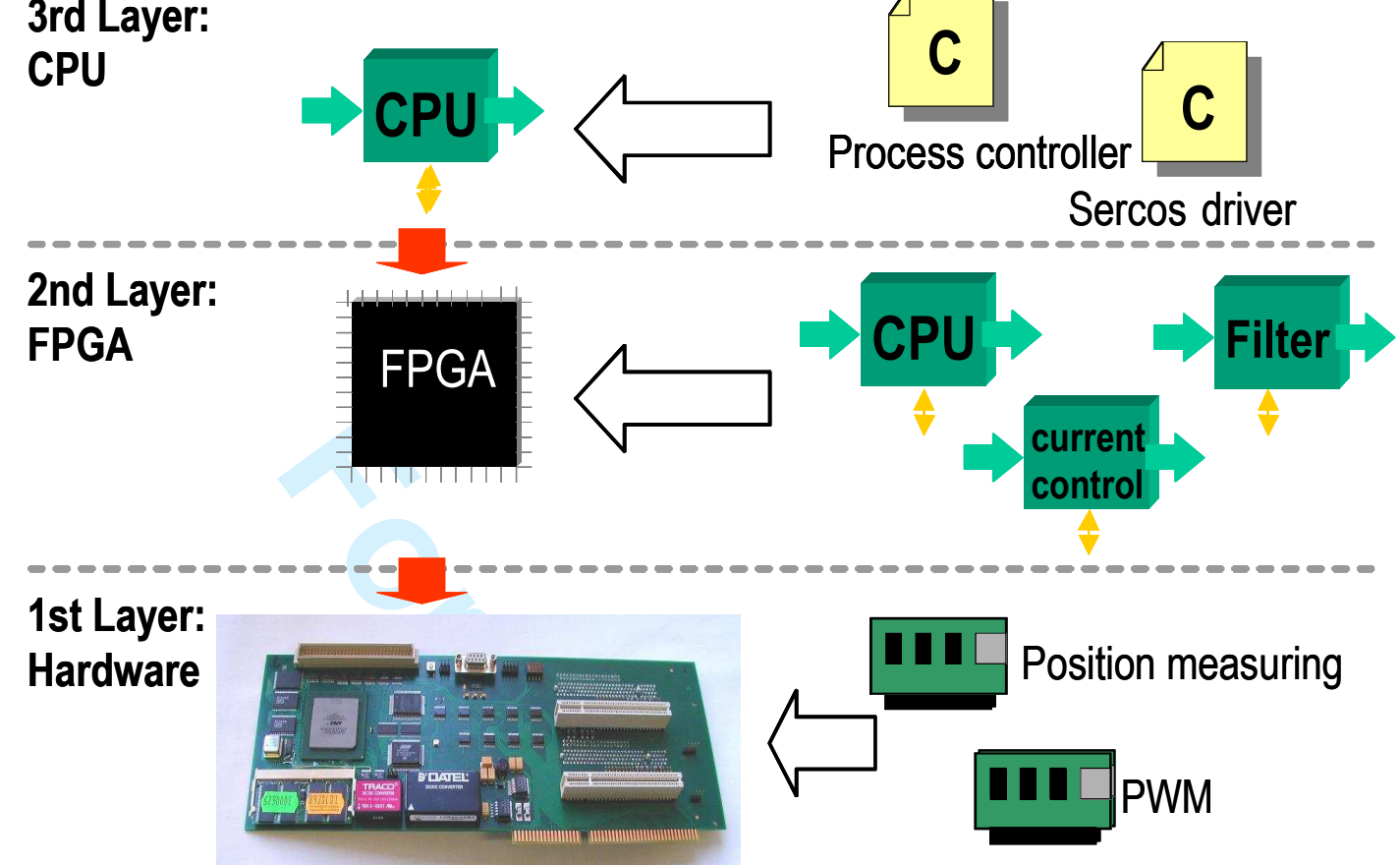

